\title{
Superconductivity and Spin Density Wave (SDW) in
}

\section{$\mathrm{NaFe}_{1-\mathrm{x}} \mathrm{Co}_{\mathrm{x}} \mathrm{As}$}

\author{
Haftu Brhane \\ Department of Physics, \\ College of Natural and Computational Sciences, \\ Haramaya University, Dire- Dawa, \\ Ethiopia
}

\author{
Amarendra Rajput \\ Department of Physics, \\ College of Natural and Computational Sciences, \\ Haramaya University, Dire- Dawa, \\ Ethiopia
}

\begin{abstract}
A model is presented utilizing a Hamiltonian with equal spin singlet and triplet pairings based on quantum field theory and green function formalism, to show the correlation between the superconducting and spin density wave (SDW) order parameters. The model exhibits a distinct possibility of the coexistence of superconductivity and long-range magnetic phase, which are two usually incompatible cooperative phenomena. The work is motivated by the recent experimental evidences on high- $\mathrm{T}_{\mathrm{C}}$ superconductivity in the FeAs-based superconductors. The theoretical results are then applied to show the coexistence of superconductivity and spin density wave (SDW) in $\mathrm{NaFe}_{1-\mathrm{x}} \mathrm{Co}_{\mathrm{x}} \mathrm{As}$.
\end{abstract}

Keywords: Retarded double time green's function formalism, Spin singlet and triplet state, superconductivity and spin density wave.

\section{INTRODUCTION}

Since the discovery of superconductivity in quaternary pnictide-oxides with critical temperatures $\left(\mathrm{T}_{\mathrm{C}}\right)$ up to $55 \mathrm{~K}$, a lot of tremendous interest has been generated in the study of co-existence of the two cooperative phenomena of superconductivity and magnetism. After first reports on superconductivity in undoped LaNiPO $[1,2]$ below $5 \mathrm{~K}$, the breakthrough was the discovery of the phenomena at $\mathrm{T}_{\mathrm{C}}$ $26 \mathrm{~K}$ in the F-doped arsenide LaO1-xFxFeAs system [3].

In addition to this several groups reported an increase of Tc values by replacing La with smaller-size rare-earth ions like $\mathrm{CeO}_{1-} \mathrm{xF}_{\mathrm{x}} \mathrm{FeAs}$ [4], and samarium-arsenide oxides $\mathrm{Sm}\left(\mathrm{O}_{1}\right.$ $\left.{ }_{\mathrm{x}} \mathrm{F}_{\mathrm{x}}\right) \mathrm{Fe}$ As with a critical temperature $\mathrm{T}_{\mathrm{c}}$ of $55 \mathrm{~K}[5,6]$. The iron based superconductors promise interesting physics and applications. While the interplay of superconductivity and magnetism, as well as their mechanisms remain the issues of active studies, one thing in FeSC riddle is clear, that it is the complex multi-band electronic structure of these compounds that determines their rich and puzzling properties. What is important and captivating is that this complexity seems to play a positive role in the struggle for understanding the FeSC physics and also for search of the materials with higher $T_{c}$ [7].

The FeSC is quite promising for applications. Having much higher $\mathrm{H}_{\mathrm{c}}$ than cuprates and high isotropic critical currents [8], they are attractive for electrical power and magnet applications, while the coexistence of magnetism and superconductivity makes them interesting for spintronics [9]. All the compounds share similar electronic band structure in which the electronic states at the Fermi level are occupied predominantly by the $\mathrm{Fe} 3 \mathrm{~d}$ electrons [7].

Scanning tunnelling microscopy studies of the local electronic structure of an underdoped $\mathrm{NaFe}_{1-\mathrm{x}} \mathrm{Co}_{\mathrm{x}} \mathrm{As}$ near the SDW and SC phase boundary. Spatially resolved spectroscopy directly reveals both SDW and SC gap features at the same atomic location, providing compelling evidence for the microscopic coexistence of the two phases. The strengths of SDW and SC features are shown to anticorrelate with each other, indicating the competition of the two orders. The underlying physical picture is that Cooper pairing in the iron pnictides can occur when portions of the Fermi surface (FS) are already gapped by the SDW order [10].

The above exciting discovery stimulated a lot of interest in the study of coexistence of superconductivity and magnetism. The proximity of the superconductivity state to the spin density wave phase in the phase diagram implies that the interplay between the magnetism and superconductivity might play an important role in understanding the pairing mechanism and other physical properties of the iron-based superconductors. It is generally believed that the magnetic couplings between the itinerant electrons and/or between the itinerant electron and local spin are essential to both spin density wave instability and superconductivity. Besides other experimental and theoretical findings, especially the antiferromagnetic ground state and the SDW anomaly of $\mathrm{LaFeAsO}$ strongly suggest that, the pairing mechanism of the electrons is likely to be connected with spin fluctuations, as it has been assumed for the cuprates [11].

In many high $\mathrm{T}_{\mathrm{C}}$ superconductors, superconducting mechanism is attributed to strong coulomb interactions of the electrons in the system, which can also be the cause for the appearance of SDW state and this suggests the existence of competition between the two states [12]. The properties of unconventional triplet superconductivity and SDW with an emphasis on the analysis of their order parameters are reviewed.

The relation between the superconducting and spindensity-wave (SDW) order is a central topic in current research on the FeAs-based high $\mathrm{T}_{\mathrm{C}}$ superconductors. So, in this paper, we start with a model Hamiltonian which incorporates the BCS theory for iron pnictide superconductors $\mathrm{NaFe}_{1-\mathrm{x}} \mathrm{Co}_{\mathrm{x}} \mathrm{As}$, to examine the coexistence of superconductivity and spin density wave.

\section{MODEL HAMILTONIAN OF THE SYSTEM}

The purpose of this work is to study theoretically the co-existence of spin density wave and superconductivity 
properties in the compound $\mathrm{NaFe}_{1-\mathrm{x}} \mathrm{Co}_{\mathrm{x}} \mathrm{As}$ in general and to find expression for transition temperature and order parameter in particular. For this purpose, we tried to find the mathematical expression for the superconducting critical temperature $\left(T_{c}\right)$, superconducting order parameter $\left(\Delta_{\mathrm{sc}}\right)$ the magnetic order parameter (M) and SDW transition temperature $\left(\mathrm{T}_{\mathrm{SDW}}\right)$. Within the framework of the BCS model, the model of the Hamiltonian for coexistence SDW and superconductivity in the compound can be express as:

$$
\begin{aligned}
H=\sum_{p \sigma} \epsilon_{p} a_{p \sigma}^{\dagger} \hat{a}_{p \sigma} & +M \sum_{p}\left(\hat{a}_{p+q \uparrow}^{\dagger} \hat{a}_{-p \downarrow}+\hat{a}_{-p \downarrow}^{\dagger} \hat{a}_{p+q \uparrow}\right) \\
& +\Delta_{\mathrm{SC}} \sum_{p}\left(\hat{a}_{p \uparrow}^{\dagger} a_{-p \downarrow}^{\dagger}+\hat{a}_{-p \downarrow} a_{p \uparrow}\right)
\end{aligned}
$$

Where $\left(\hat{a}_{p \sigma}^{\dagger} a_{p \sigma}\right)$ are the creation (annihilation) operators of an electron having the wave number $p$ and $\operatorname{spin} \sigma$. Whereas $\left(\Delta_{\mathrm{SC}}\right)$ superconducting order parameter and (M) SDW order parameters. The Hamiltonian in (1) will be used to determine the equations of motion in terms of the Green function.

\section{COUPLING OF SDW AND SUPERCONDUCTING ORDER PARAMETERS}

The Double time dependent Green's function equal to the change of the average value of some dynamic quantity by the time $t$ and useful because they can be used to describe the effect of retarded interactions and all quantities of physical interest can be derived from them. To get the equation of motion we use the double-time temperature dependent retarded Green function is given by Zubarev [13]:

$$
\begin{gathered}
G_{r}\left(t-t^{\prime}\right) \equiv \ll \hat{A}(t) ; \hat{B}\left(t^{\prime}\right) \gg \\
\text { or } G_{r}\left(t, t^{\prime}\right)=-i \theta\left(t-t^{\prime}\right)<\left[\hat{A}(t), \hat{B}\left(t^{\prime}\right)>\right.
\end{gathered}
$$

Where $\hat{A}$ and $\hat{B}$ are Heisenberg operators and $\theta\left(t-t^{\prime}\right)$ is the Heaviside step function. Now, using Dirac delta function and Heisenberg operators, we can write as;

$$
\begin{aligned}
& i \frac{d}{d t} G_{r}\left(t-t^{\prime}\right)=\delta\left(t-t^{\prime}\right)<\left[\hat{A}(t), \hat{B}\left(t^{\prime}\right)\right]>+ \\
&\left.<<[\hat{A}(t), H], \hat{B}\left(t^{\prime}\right)\right]>>
\end{aligned}
$$

The Fourier transformation $G_{r}(\omega)$ is given by

$$
G_{r}\left(t-t^{\prime}\right)=\int G_{r}(\omega) \exp \left[-i \omega\left(t-t^{\prime}\right)\right] d \omega
$$

Taking the Fourier transform we get:

$$
\begin{aligned}
\omega G_{r}(\omega)=<\left[\hat{A}(t), \hat{B}\left(t^{\prime}\right)\right] & >_{\omega} \\
& \left.+\ll[\hat{A}(t), H], \hat{B}\left(t^{\prime}\right)\right] \gg_{\omega}
\end{aligned}
$$

From (4), it follows that

$$
\omega \ll \hat{a}_{\kappa \uparrow}^{\dagger}, \hat{a}^{\dagger}{ }_{-\kappa \downarrow} \gg=\left\langle\left[\hat{a}_{\kappa \uparrow}^{\dagger}, H\right], \hat{a}_{-\kappa \downarrow}^{\dagger} \gg\right.
$$

where the anti-commutation relation,

$$
\left\{\hat{a}_{\kappa \sigma}, \hat{a}^{\dagger}{ }_{k^{\prime} \sigma^{\prime}}\right\}=\delta_{k k^{\prime}} \delta_{\sigma \sigma^{\prime}}
$$

has been used. To derive an expression for $\left\langle\hat{a}^{\dagger}{ }_{\kappa \uparrow}, \hat{a}^{\dagger}{ }_{-\kappa \downarrow}\right\rangle$, we have calculate the commutator $\left[\hat{a}^{\dagger}{ }_{\kappa \uparrow}, H\right]$, using (1) and using the identities and

$$
\begin{aligned}
& {[A, B C]=\{A, B\} C-B\{A, C\}} \\
& \{A, C\} B
\end{aligned} \text { (7) } \quad \text { and } \quad[A B, C]=A\{B, C\}-
$$

Solving the commutator in eq.(5) by using the Hamiltonian in eq.(1), we get

$$
\begin{aligned}
& {\left[\hat{a}^{\dagger}{ }_{\kappa \uparrow}, \sum_{p \sigma} \epsilon_{p} \hat{a}_{p \sigma}^{\dagger} \hat{a}_{p \sigma}\right]=\sum_{p \sigma} \epsilon_{p}\left[\hat{a}^{\dagger}{ }_{\kappa \uparrow}, \hat{a}^{\dagger}{ }_{p \sigma} \hat{a}_{p \sigma}\right]} \\
& =\sum_{p \sigma} \epsilon_{p}\left(\left\{\hat{a}^{\dagger}{ }_{\kappa \uparrow}, a_{p \sigma}^{\dagger}\right\} a_{p \sigma}-a_{p \sigma}^{\dagger}\left\{\hat{a}^{\dagger}{ }_{\kappa \uparrow}, \hat{a}_{p \sigma}\right\}\right. \\
& {\left[\hat{a}^{\dagger}{ }_{\kappa \uparrow}, \sum_{p \sigma} \epsilon_{p} \hat{a}_{p \sigma}^{\dagger} \hat{a}_{p \sigma}\right]=-\epsilon_{\kappa} \hat{a}^{\dagger}{ }_{\kappa \uparrow}}
\end{aligned}
$$

After some lengthy but straightforward calculations; we arrive at the following results:

$$
\begin{gathered}
{\left[\hat{a}_{\kappa \uparrow}^{\dagger}, M \sum_{\kappa}\left(\hat{a}_{\kappa+q \uparrow}^{\dagger} \hat{a}_{-\kappa \downarrow}+a_{-\kappa \downarrow}^{\dagger} \hat{a}_{\kappa+q \uparrow}\right)\right]=-M \hat{a}_{\kappa-q \downarrow}^{\dagger}(8 b} \\
{\left[\hat{a}^{\dagger}{ }_{\kappa \uparrow}, \Delta_{\mathrm{SC}} \sum_{p}\left(a_{p \uparrow}^{\dagger} a_{-p \downarrow}^{\dagger}+\hat{a}_{-p \downarrow} \hat{a}_{p \uparrow}\right)\right]=-\Delta_{\mathrm{SC}} \hat{a}_{-k \downarrow}}
\end{gathered}
$$

Substituting (8) in to (5), we get

$$
\begin{aligned}
& \omega \ll \hat{a}^{\dagger}{ }_{\kappa \uparrow}, \hat{a}^{\dagger}{ }_{-\kappa \downarrow} \gg=-\epsilon_{\kappa} \ll \hat{a}^{\dagger}{ }_{\kappa \uparrow}, \hat{a}^{\dagger}{ }_{-\kappa \downarrow} \gg-M \\
& \ll \hat{a}^{\dagger}{ }_{\kappa-q \downarrow}, \hat{a}^{\dagger}{ }_{-\kappa \downarrow} \gg-\Delta_{\mathrm{SC}} \ll \hat{a}_{-k \downarrow}, \hat{a}^{\dagger}{ }_{-\kappa \downarrow} \\
& \gg \\
&\left(\omega+\epsilon_{\kappa}\right) \ll \hat{a}^{\dagger}{ }_{\kappa \uparrow}, \hat{a}^{\dagger}{ }_{-\kappa \downarrow} \gg=-M \ll \hat{a}^{\dagger}{ }_{\kappa-q \downarrow}, \hat{a}^{\dagger}{ }_{-\kappa \downarrow} \gg-\Delta_{\mathrm{SC}} \\
& \ll
\end{aligned}
$$

The equation of motion for the correlation $\ll \hat{a}_{\kappa-q \downarrow}^{\dagger}, \hat{a}_{-\kappa \downarrow}^{\dagger} \gg$ in (9) can be described as:

$$
\begin{aligned}
& \omega \ll \hat{a}_{\kappa-q \downarrow}^{\dagger}, \hat{a}_{-\kappa \downarrow}^{\dagger} \gg=\delta_{k k^{\prime}}+\ll\left[\hat{a}_{\kappa-q \downarrow}^{\dagger}, H\right], \hat{a}_{-\kappa \downarrow}^{\dagger} \gg \\
& \omega \ll \hat{a}_{\kappa-q \downarrow}^{\dagger}, \hat{a}_{-\kappa \downarrow}^{\dagger} \gg=\ll\left[\hat{a}_{\kappa-q \downarrow}^{\dagger}, H\right], \hat{a}_{-\kappa \downarrow}^{\dagger} \gg \quad(10)
\end{aligned}
$$

Evaluating the commutator in eq.(10) using Hamiltonian:

$$
\begin{gathered}
{\left[\hat{a}^{\dagger}{ }_{\kappa-q \downarrow}, \sum_{p \sigma} \epsilon_{p} \hat{a}_{p \sigma}^{\dagger} \hat{a}_{p \sigma}\right]=\sum_{p \sigma} \epsilon_{p}\left[\hat{a}^{\dagger}{ }_{\kappa-q \downarrow}, \hat{a}_{p \sigma}^{\dagger}{ }_{p \sigma} \hat{a}_{p \sigma}\right]} \\
=\sum_{p \sigma} \epsilon_{p}\left(\left\{\hat{a}^{\dagger}{ }_{\kappa-q \downarrow}, \hat{a}_{p \sigma}^{\dagger}\right\} \hat{a}_{p \sigma}-\hat{a}_{p \sigma}^{\dagger}\left\{\hat{a}^{\dagger}{ }_{\kappa-q \downarrow}, \hat{a}_{p \sigma}\right\}\right. \\
{\left[\hat{a}^{\dagger}{ }_{\kappa-q \downarrow}, \sum_{p \sigma} \epsilon_{p} \hat{a}_{p \sigma}^{\dagger} \hat{a}_{p \sigma}\right]=-\epsilon_{\kappa-q} \hat{a}_{\kappa-q \downarrow}^{\dagger}{ }_{\kappa-q}}
\end{gathered}
$$

After some lengthy but straightforward calculations; we arrive at the following results:

$$
\begin{gathered}
{\left[\hat{a}^{\dagger}{ }_{\kappa-q \downarrow}, M \sum_{\kappa}\left(\hat{a}_{\kappa+q \uparrow}^{\dagger} a_{-\kappa \downarrow}+a_{-\kappa \downarrow}^{\dagger} \hat{a}_{\kappa+q \uparrow}\right)\right]=-M \hat{a}^{\dagger}{ }_{\kappa \uparrow}} \\
{\left[\hat{a}^{\dagger}{ }_{\kappa-q \downarrow}, \Delta_{\mathrm{SC}} \sum_{p}\left(a_{p \uparrow}^{\dagger} \hat{a}_{-p \downarrow}^{\dagger}+a_{-p \downarrow} a_{p \uparrow}\right)\right]=\Delta_{\mathrm{SC}} \hat{a}_{-k+q \downarrow}}
\end{gathered}
$$

Substituting (11) in to (10), we get

$$
\begin{aligned}
& \omega \ll \hat{a}_{\kappa-q \downarrow}^{\dagger}, \hat{a}^{\dagger}{ }_{-\kappa \downarrow} \gg=-\epsilon_{\kappa-q} \ll \hat{a}^{\dagger}{ }_{\kappa-q \downarrow}, \hat{a}^{\dagger}{ }_{-\kappa \downarrow} \gg-M \\
& \ll \hat{a}^{\dagger}{ }_{\kappa \uparrow}, \hat{a}^{\dagger}{ }_{-\kappa \downarrow} \gg+\Delta_{\mathrm{SC}} \ll \hat{a}_{-k+q \downarrow}, \hat{a}^{\dagger}{ }_{-\kappa \downarrow} \\
& \gg \\
& \begin{array}{r}
\left(\omega+\epsilon_{\kappa-q}\right) \ll \hat{a}^{\dagger}{ }_{\kappa-q \downarrow}, \hat{a}^{\dagger}{ }_{-\kappa \downarrow} \gg=-M \ll \hat{a}^{\dagger}{ }_{\kappa \uparrow}, \hat{a}_{-\kappa \downarrow}^{\dagger} \\
\gg+\Delta_{\mathrm{SC}} \ll \hat{a}_{-k+q \downarrow}, \hat{a}^{\dagger}{ }_{-\kappa \downarrow} \gg \quad(12)
\end{array}
\end{aligned}
$$

Similarly as we did in the above the equation of motion for the correlation $\ll \hat{a}_{-\kappa+q \uparrow}, \hat{a}_{-\kappa \downarrow}^{\dagger} \gg$ and $\hat{a}_{-\kappa \downarrow}, \hat{a}^{\dagger}{ }_{-\kappa \downarrow} \gg$ is given by:

$$
\begin{aligned}
\omega \ll \hat{a}_{-\kappa+q \uparrow}, \hat{a}^{\dagger}-\kappa \downarrow & \gg=\epsilon_{-\kappa+q} \ll \hat{a}_{-\kappa+q \uparrow}, \hat{a}^{\dagger}{ }_{-\kappa \downarrow} \gg+M \\
& \ll \hat{a}_{-\kappa \downarrow}, \hat{a}^{\dagger}-{ }_{-\kappa \downarrow} \gg+\Delta_{\mathrm{SC}} \ll \hat{a}^{\dagger}{ }_{\kappa-q \downarrow}, \hat{a}^{\dagger}{ }_{-\kappa \downarrow} \\
& \gg
\end{aligned}
$$




$$
\begin{array}{r}
\left(\omega-\epsilon_{-\kappa+q}\right) \ll \hat{a}_{-\kappa+q \uparrow}, \hat{a}^{\dagger}{ }_{-\kappa \downarrow} \gg=M \ll \hat{a}_{-\kappa \downarrow}, \hat{a}^{\dagger}{ }_{-\kappa \downarrow} \\
\gg+\Delta_{\mathrm{SC}} \ll \hat{a}^{\dagger}{ }_{\kappa-q \downarrow}, \hat{a}^{\dagger}{ }_{-\kappa \downarrow} \gg(13)
\end{array}
$$

and

$$
\begin{array}{r}
\left(\omega-\epsilon_{-\kappa}\right) \ll \hat{a}_{-\kappa \downarrow}, \hat{a}^{\dagger}{ }_{-\kappa \downarrow} \gg=1+M \ll \hat{a}_{-\kappa+q \uparrow}, \hat{a}^{\dagger}{ }_{-\kappa \downarrow} \\
\gg-\Delta_{\mathrm{SC}} \ll \hat{a}^{\dagger}{ }_{\kappa \uparrow}, \hat{a}^{\dagger}{ }_{-\kappa \downarrow} \gg
\end{array}
$$

From eq. (12), we obtain:

$$
\begin{aligned}
\left\langle\left\langle\hat{a}^{\dagger}{ }_{\kappa-q \downarrow}, \hat{a}^{\dagger}{ }_{-\kappa \downarrow} \gg\right.\right. & =\frac{-M \ll \hat{a}_{\kappa \uparrow}^{\dagger}, \hat{a}_{-\kappa \downarrow}^{\dagger} \gg}{\left(\omega+\epsilon_{\kappa-q}\right)} \\
& +\frac{\Delta_{\mathrm{SC}} \ll\left\langle\hat{a}_{-k+q \downarrow}, \hat{a}^{\dagger}{ }_{-\kappa \downarrow} \gg\right.}{\left(\omega+\epsilon_{\kappa-q}\right)}
\end{aligned}
$$

And from eq. (14):

$$
\begin{aligned}
\left\langle\hat{a}_{-\kappa \downarrow}, \hat{a}_{-\kappa \downarrow}^{\dagger} \gg=\right. & \frac{1}{\left(\omega-\epsilon_{-\kappa}\right)}+\frac{M \ll \hat{a}_{-\kappa+q \uparrow}, \hat{a}^{\dagger}{ }_{-\kappa \downarrow} \gg}{\left(\omega-\epsilon_{-\kappa}\right)} \\
& -\frac{\Delta_{\mathrm{SC}} \ll \hat{a}^{\dagger}{ }_{\kappa \uparrow}, \hat{a}^{\dagger}{ }_{-\kappa \downarrow} \gg}{\left(\omega-\epsilon_{-\kappa}\right)} \text { (16) }
\end{aligned}
$$

Plugging eq.(15) and (16) in (9), yields:

$$
\begin{gathered}
\left(\omega+\epsilon_{\kappa}-\frac{M^{2}}{\left(\omega+\epsilon_{\kappa-q}\right)}-\frac{\Delta_{\mathrm{SC}}^{2}}{\left(\omega-\epsilon_{-\kappa}\right)}\right) \ll \hat{a}_{\kappa \uparrow}^{\dagger}, \hat{a}^{\dagger}{ }_{-\kappa \downarrow} \gg \\
=-\frac{\Delta_{\mathrm{SC}}}{\left(\omega-\epsilon_{-\kappa}\right)}- \\
\left(\frac{M \Delta_{\mathrm{SC}}}{\left(\omega+\epsilon_{\kappa-q}\right)}+\frac{M \Delta_{\mathrm{SC}}}{\left(\omega-\epsilon_{-\kappa}\right)}\right) \ll \hat{a}_{-k+q \downarrow}, \hat{a}_{-\kappa \downarrow}^{\dagger} \gg \text { (17) }
\end{gathered}
$$

And insert eq. (15) and (16) in (13), we have:

$$
\begin{gathered}
\left(\left(\omega-\epsilon_{-\kappa+q}\right)-\frac{M^{2}}{\left(\omega-\epsilon_{-\kappa}\right)}-\frac{\Delta_{\mathrm{SC}}{ }^{2}}{\left(\omega+\epsilon_{\kappa-q}\right)}\right) \ll \hat{a}_{-\kappa \downarrow}, \hat{a}_{-\kappa \downarrow}^{\dagger} \gg= \\
\gg=-\frac{M}{\left(\omega-\epsilon_{-\kappa}\right)}- \\
\left(\frac{M \Delta_{\mathrm{SC}}}{\left(\omega-\epsilon_{-\kappa}\right)}+\frac{M \Delta_{\mathrm{SC}}}{\left(\omega+\epsilon_{k-q}\right)}\right) \ll \hat{a}_{\kappa \uparrow}^{\dagger}, \hat{a}_{-\kappa \downarrow}^{\dagger} \gg(18)
\end{gathered}
$$

Applying nesting condition $\epsilon_{\kappa}=-\epsilon_{\kappa \pm q}, \epsilon_{-\kappa}=\epsilon_{\kappa \mp q}$ and use approximation, $\epsilon_{\kappa}=\epsilon_{-\kappa}$; eq.(17) and (14) becomes:

$$
\begin{aligned}
& \left(\omega+\epsilon_{\kappa}-\frac{M^{2}}{\left(\omega-\epsilon_{\kappa}\right)}-\frac{\Delta_{\mathrm{SC}^{2}}}{\left(\omega-\epsilon_{k}\right)}\right) \ll \hat{a}^{\dagger}{ }_{\kappa \uparrow}, \hat{a}_{-\kappa \downarrow}^{\dagger} \gg \\
& =-\frac{\Delta_{\mathrm{SC}}}{\left(\omega-\epsilon_{\kappa}\right)}- \\
& \left(\frac{M \Delta_{\mathrm{SC}}}{\left(\omega-\epsilon_{\kappa}\right)}+\frac{M \Delta_{\mathrm{SC}}}{\left(\omega-\epsilon_{\kappa}\right)}\right) \ll \hat{a}_{-k+q \downarrow}, \hat{a}^{\dagger}{ }_{-\kappa \downarrow} \gg
\end{aligned}
$$

And

$$
\begin{aligned}
\left(\left(\omega+\epsilon_{k}\right)-\frac{M^{2}}{\left(\omega-\epsilon_{k}\right)}\right. & \left.-\frac{\Delta_{\mathrm{SC}}{ }^{2}}{\left(\omega-\epsilon_{\kappa}\right)}\right) \ll \hat{a}_{-\kappa \downarrow}, \hat{a}_{-\kappa \downarrow}^{\dagger} \gg= \\
& =-\frac{M}{\left(\omega-\epsilon_{k}\right)}-\left(\frac{M \Delta_{\mathrm{SC}}}{\left(\omega-\epsilon_{k}\right)}+\frac{M \Delta_{\mathrm{SC}}}{\left(\omega-\epsilon_{k}\right)}\right) \\
& \ll \hat{a}^{\dagger}{ }_{\kappa \uparrow}, \hat{a}_{-\kappa \downarrow}^{\dagger} \gg
\end{aligned}
$$

Let $x=\omega+\epsilon_{k}$ and $y=\omega-\epsilon_{k}$

Then eq. (19) and (20) respectively becomes:

$$
\begin{aligned}
{\left[x y-M^{2}-\Delta_{\mathrm{SC}}{ }^{2}\right] } & \ll \hat{a}^{\dagger}{ }_{\kappa \uparrow}, \hat{a}^{\dagger}{ }_{-\kappa \downarrow} \gg=-\Delta_{\mathrm{SC}}-2 M \Delta_{\mathrm{SC}} \\
& \ll \hat{a}_{-k+q \uparrow}, \hat{a}^{\dagger}{ }_{-\kappa \downarrow} \gg
\end{aligned}
$$

$$
\begin{aligned}
{\left[x y-M^{2}-\Delta_{\mathrm{SC}}{ }^{2}\right] } & \ll \hat{a}_{-k+q \downarrow}, \hat{a}^{\dagger}{ }_{-\kappa \downarrow} \gg=-\Delta_{\mathrm{SC}}-2 M \Delta_{\mathrm{SC}} \\
& \ll \hat{a}^{\dagger}{ }_{\kappa \uparrow}, \hat{a}^{\dagger}{ }_{-\kappa \downarrow} \gg(22)
\end{aligned}
$$

Finally we can express:

$$
\begin{aligned}
\ll \hat{a}_{\kappa \uparrow}^{\dagger}, \hat{a}^{\dagger}{ }_{-\kappa \downarrow} \gg= & \frac{-1 / 2\left(\Delta_{\mathrm{SC}}+M\right)}{\omega^{2}-\epsilon_{\kappa}{ }^{2}-\left(\Delta_{\mathrm{SC}}+M\right)^{2}} \\
& +\frac{-1 / 2\left(\Delta_{\mathrm{SC}}-M\right)}{\omega^{2}--\epsilon_{\kappa}{ }^{2}-\left(\Delta_{\mathrm{SC}}-M\right)^{2}}
\end{aligned}
$$

Using the expression $\omega \rightarrow i \omega_{n}, \Delta_{j}(k)=-(-1)^{j} M$, where $\Delta_{j}(k)$ is effective order parameter and the Matsubara's frequency, we can write eq. (23) as:

$$
\begin{aligned}
& \ll \hat{a}^{\dagger}{ }_{\kappa \uparrow}, \hat{a}^{\dagger}{ }_{-\kappa \downarrow} \gg \\
& =\frac{1}{2} \sum_{j=1,2} \frac{\beta^{2} \Delta_{j}(k)}{(2 n+1)^{2} \pi^{2}+\beta^{2}\left(\epsilon_{\kappa}^{2}+\Delta_{j}^{2}(k)\right)}
\end{aligned}
$$

To take into account the temperature dependence of order parameters, we shall write as:

$$
\begin{aligned}
& \Delta_{\mathrm{SC}}=\frac{V}{\beta} \sum_{\kappa, n} \ll \hat{a}_{\kappa \uparrow}^{\dagger}, \hat{a}_{-\kappa \downarrow}^{\dagger} \gg \\
& M=\frac{U}{\beta} \sum_{k} \ll\left\langle\hat{a}^{\dagger}{ }_{\kappa \uparrow}, \hat{a}_{k-q \downarrow} \gg\right.
\end{aligned}
$$

Where $\beta=\frac{1}{K T}$

Using eq.(24) into eq.(25), we obtain

$$
\Delta_{\mathrm{SC}}=\frac{V}{2} \sum_{\kappa, n, j=1,2} \frac{\beta \Delta_{j}(k)}{(2 n+1)^{2} \pi^{2}+\beta^{2}\left(\epsilon_{\kappa}^{2}+\Delta_{j}^{2}(k)\right)}
$$

Let us use

$$
\gamma=\beta\left(\epsilon_{\kappa}^{2}+\Delta_{j}^{2}(k)\right)^{1 / 2}
$$

and

$$
\sum_{-\infty}^{\infty} \frac{1}{(2 n+1)^{2} \pi^{2}+\gamma}=\frac{\tanh \gamma / 2}{2 \gamma}
$$

Plugging eq.(28) and eq.(29) in eq.(27), we get:

$$
\Delta_{\mathrm{SC}}=\frac{V}{4} \sum_{j=1,2} \frac{\Delta_{j}(k) \tanh \frac{\beta}{2}\left(\epsilon_{\kappa}^{2}+\Delta_{j}^{2}(k)\right)^{1 / 2}}{\left(\epsilon_{\kappa}^{2}+\Delta_{j}^{2}(k)\right)^{1 / 2}}
$$

For mathematical convenience, we replace the summation in (27) by integration. Thus

$$
\sum_{k} \equiv \int_{-\hbar \omega_{b}}^{\hbar \omega_{b}} N(0) d \epsilon_{\kappa}
$$

where $N(0)$ is the density of states at the Fermi level.

The density of state $N(0)=N(2)_{1}+N(0)_{2}$

Assume $N(2)_{1}=N(0)_{2}$ this implies that $N(0)_{2}=N(0) / 2$. For $\mathrm{j}=2$ :

$$
\begin{aligned}
& \Delta_{\mathrm{SC}} \\
& =\alpha \int_{0}^{\hbar \omega_{b}}\left(\Delta_{\mathrm{SC}}-M\right) \frac{\tanh \frac{\beta}{2}\left(\epsilon_{\kappa}{ }^{2}+\left(\Delta_{\mathrm{SC}}-M\right)^{2}\right)^{1 / 2}}{\left(\epsilon_{\kappa}{ }^{2}+\left(\Delta_{\mathrm{SC}}-M\right)^{2}\right)^{1 / 2}} d \epsilon_{\kappa} 31
\end{aligned}
$$

Where $\alpha=N(0)_{2} V$ 
Finally we can write eq.(31) as:

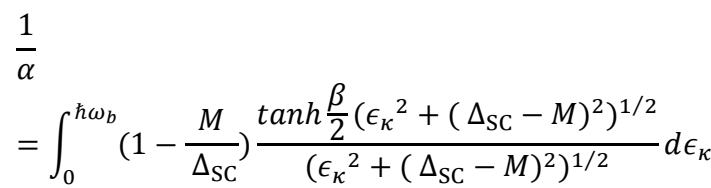

From (32), it clearly follows that the order parameters $\Delta_{\mathrm{SC}}$ and $M \quad$, for superconductivity and SDW are interdependent.

We now consider the equations of motion for SDW, we can write,

$$
\omega \ll \hat{a}^{\dagger}{ }_{\kappa \uparrow}, \hat{a}_{k-q \downarrow} \gg=\delta_{K k^{\prime}}+\ll\left[\hat{a}^{\dagger}{ }_{\kappa \uparrow}, H\right], \hat{a}_{k-q \downarrow} \gg
$$

Doing a lot as we did in the above, we finally get:

$$
\begin{aligned}
& \left(\omega+\epsilon_{\kappa}\right) \ll \hat{a}^{\dagger}{ }_{\kappa \uparrow}, \hat{a}_{k-q \downarrow} \gg=-M \ll \hat{a}^{\dagger}{ }_{\kappa-q \downarrow}, \hat{a}_{k-q \downarrow} \gg-\Delta_{\mathrm{SC}} \\
& \ll \hat{a}_{-k \downarrow}, \hat{a}_{k-q \downarrow} \gg \\
& \left(\omega+\epsilon_{\kappa-q}\right) \ll \hat{a}^{\dagger}{ }_{\kappa-q \downarrow}, \hat{a}_{k-q \downarrow} \gg=1-M \ll \hat{a}^{\dagger}{ }_{\kappa \uparrow}, \hat{a}_{k-q \downarrow} \\
& \gg+\Delta_{\mathrm{SC}} \ll \hat{a}_{-k+q \uparrow}, \hat{a}_{k-q \downarrow} \gg
\end{aligned}
$$

And

$$
\begin{gathered}
\left(\omega-\epsilon_{-\kappa}\right) \ll \hat{a}_{-k \downarrow}, \hat{a}_{k-q \downarrow} \gg=M \ll \hat{a}_{-k+q \uparrow}, \hat{a}_{k-q \downarrow} \gg-\Delta_{\mathrm{SC}} \\
\ll \hat{a}^{\dagger}{ }_{\kappa \uparrow}, \hat{a}_{k-q \downarrow} \gg
\end{gathered}
$$

Doing a lot as we did in the previous, we finally get:

$$
\begin{aligned}
& \ll \hat{a}^{\dagger}{ }_{\kappa \uparrow}, \hat{a}_{k-q \downarrow} \gg \\
& =\frac{1}{2} \sum_{j=1,2} \frac{(-1)^{j} \beta^{2} \Delta_{j}(k)}{(2 n+1)^{2} \pi^{2}+\beta^{2}\left(\epsilon_{k}^{2}+\Delta_{j}^{2}(k)\right)}
\end{aligned}
$$

Using eq.(38) in to eq.(26), the SDW order Parameter M is given by:

$$
M=-\frac{U}{2} \sum_{k, n, j=1} \frac{\beta \Delta_{j}(k)}{(2 n+1)^{2} \pi^{2}+\beta^{2}\left(\epsilon_{\kappa}^{2}+\Delta_{j}^{2}(k)\right)}
$$

Or

$$
M=\frac{-U}{4} \sum_{j=1} \frac{\Delta_{j}(k) \tanh \frac{\beta}{2}\left(\epsilon_{\kappa}^{2}+\Delta_{j}^{2}(k)\right)^{1 / 2}}{\left(\epsilon_{\kappa}^{2}+\Delta_{j}^{2}(k)\right)^{1 / 2}}
$$

So, finally we get:

$$
M=-\alpha_{j} \Delta_{j} \int_{0}^{\hbar \omega_{b} \tanh \frac{\beta}{2}\left(\epsilon_{\kappa}^{2}+\Delta_{j}^{2}(k)\right)^{1 / 2}}{\left(\epsilon_{\kappa}^{2}+\Delta_{j}^{2}(k)\right)^{1 / 2}}_{\epsilon}
$$

From (41), it is again evident that the order parameters $\Delta_{\mathrm{SC}}$ and $M \quad$, for superconductivity and SDW are interdependent, as was the case from (32).

It is, therefore, possible that in some temperature interval, SDW and superconductivity can co-exist, although one phase has a tendency to suppress the critical temperature and the order parameter of the other phase.

\section{DEPENDENCE OF THE MAGNETIC ORDER PARAMETER ON THE TRANSITION TEMPERATURE FOR SUPERCONDUCTIVITY AND SDW}

$T \rightarrow 0 K, \beta \rightarrow \infty$

To study eq.(32), we consider the case, when

We can then replace

$$
\tanh \frac{\beta}{2}\left(\epsilon_{\kappa}^{2}+\left(\Delta_{\mathrm{SC}}-M\right)^{2}\right)^{1 / 2} \rightarrow 1
$$

In (32) and get,

$$
\frac{1}{\alpha}=\int_{0}^{\hbar \omega_{b}}\left(1-\frac{M}{\Delta_{\mathrm{SC}}}\right) \frac{1}{\left(\epsilon_{\kappa}^{2}+\left(\Delta_{\mathrm{SC}}-M\right)^{2}\right)^{1 / 2}} d \epsilon_{\kappa}
$$

Using the integral relation,

$$
\begin{array}{r}
\int \frac{y}{\sqrt{y^{2}+x^{2}}} d x=y \sin ^{-1}(x / y) \\
\frac{1}{\alpha}=\left(1-\frac{M}{\Delta_{\mathrm{SC}}}\right) \sin ^{-1} \frac{\left(\hbar \omega_{b}\right)}{\left(\Delta_{\mathrm{SC}}-M\right)}
\end{array}
$$

the above equation reduces to,

$$
\Delta_{\mathrm{SC}}-M=2 \hbar \omega_{b} \exp \left(-\frac{1}{\alpha\left(1-\frac{M}{\Delta_{\mathrm{SC}}}\right)}\right)
$$

from the BCS theory, the order parameter $\Delta_{\mathrm{SC}}$, at $\mathrm{T}=0$ for a given superconductor with transition temperature $T_{C}$ is given by

$$
2 \Delta_{\mathrm{SC}}(0)=3.53 k_{B} T_{C}
$$

using this result in (43), we obtain

$$
M=1.75 k_{B} T_{c}-2 \hbar \omega_{b} \exp \left(-\frac{1}{\alpha\left(1-\frac{M}{1.75 k_{B} T_{c}}\right)}\right)
$$

To solve (45) numerically we use Debay temperature and the interband BCS coupling constant.

$$
\text { To estimate } \alpha \text {, we consider the cas } \quad T \rightarrow T_{C}
$$
which implies, $\quad \Delta_{\mathrm{SC}} \rightarrow 0$

From (32), we then have

$$
\begin{aligned}
& \frac{1}{\alpha} \\
& =\int_{0}^{\hbar \omega_{b}} \frac{\tanh \frac{\beta}{2}\left(\epsilon_{\kappa}^{2}+\left(\Delta_{\mathrm{SC}}-M\right)^{2}\right)^{1 / 2}}{\left(\epsilon_{\kappa}^{2}+\left(\Delta_{\mathrm{SC}}-M\right)^{2}\right)^{1 / 2}} d \epsilon_{\kappa} \\
& -\int_{0}^{\hbar \omega_{b}} \frac{M}{\Delta_{\mathrm{SC}}} \frac{\tanh \frac{\beta}{2}\left(\epsilon_{\kappa}^{2}+\left(\Delta_{\mathrm{SC}}-M\right)^{2}\right)^{1 / 2}}{\left(\epsilon_{\kappa}^{2}+\left(\Delta_{\mathrm{SC}}-M\right)^{2}\right)^{1 / 2}} d \epsilon_{\kappa} \\
& \frac{1}{\alpha}=I_{1}-I_{2}
\end{aligned}
$$




$$
\begin{aligned}
& \frac{1}{\alpha} \\
& \left.=\int_{0}^{\hbar \omega_{b}} \frac{\tanh \frac{\beta}{2}\left(\epsilon_{\kappa}^{2}+\left(\Delta_{\mathrm{SC}}-M\right)^{2}\right)^{1 / 2}}{\hbar \omega_{b}} d \epsilon_{\kappa}{ }^{2}+\left(\Delta_{\mathrm{SC}}-M\right)^{2}\right)^{1 / 2} \\
& -\lim _{\Delta_{\mathrm{SC}} \rightarrow 0} \int_{0}^{\hbar \omega_{b}} \frac{M}{\Delta_{\mathrm{SC}}} \frac{\tanh \frac{\beta}{2}\left(\epsilon_{\kappa}{ }^{2}+\left(\Delta_{\mathrm{SC}}-M\right)^{2}\right)^{1 / 2}}{\left(\epsilon_{\kappa}{ }^{2}+\left(\Delta_{\mathrm{SC}}-M\right)^{2}\right)^{1 / 2}} d \epsilon_{\kappa} \\
& \text { Putting } \tau^{2}=\beta \sqrt{\epsilon_{\kappa}{ }^{2}+M^{2}} \text { and for } \Delta_{\mathrm{SC}}=0
\end{aligned}
$$

we can write

$$
\begin{array}{r}
I_{1}=\int_{0}^{\hbar \omega_{b}} \frac{\tanh \frac{\beta}{2}\left(\epsilon_{\kappa}^{2}+M^{2}\right)^{1 / 2}}{\left(\epsilon_{\kappa}^{2}+M^{2}\right)^{1 / 2}} d \epsilon_{\kappa} \\
=\int_{0}^{\hbar \omega_{b}} \frac{2}{2 \tau} \beta \tanh \tau / 2 d \epsilon_{\kappa}
\end{array}
$$

Using Laplacian's transformation with Matsuber relation result we can write,

$$
\begin{aligned}
\int_{0}^{\hbar \omega_{b}} \frac{\tanh \frac{\beta}{2}\left(\epsilon_{\kappa}{ }^{2}+M^{2}\right)^{1 / 2}}{\left(\epsilon_{\kappa}^{2}+M^{2}\right)^{1 / 2}} d \epsilon_{\kappa} \\
=\int_{0}^{\hbar \omega_{b}} \frac{\tanh \beta \epsilon_{\kappa} / 2}{\epsilon_{\kappa}} d \epsilon_{\kappa} \\
-\int_{0}^{\hbar \omega_{b}} \frac{4}{\beta} \sum_{n=0}^{\infty} \frac{M^{2}}{a^{4}\left(1+x^{2}\right)^{2}}
\end{aligned}
$$

Where $x^{2}=\frac{\epsilon_{\kappa}^{2}}{a^{2}}$ and $a=(2 n+1) \frac{\pi}{\beta}$ and using integrating by part,

$$
\begin{gathered}
\int_{0}^{\hbar \omega_{b}} \frac{\tanh \frac{\beta}{2}\left(\epsilon_{\kappa}^{2}+M^{2}\right)^{1 / 2}}{\left(\epsilon_{\kappa}^{2}+M^{2}\right)^{1 / 2}} d \epsilon_{\kappa} \\
=\left.(\ln x)(\tanh x)\right|_{0} ^{x}-\int_{0}^{x} \frac{\ln x}{\cosh ^{2} x} d x \\
-\int_{0}^{\hbar \omega_{b}} \frac{4}{\beta} \sum_{n=0}^{\infty} \frac{M^{2}}{a^{4}\left(1+x^{2}\right)^{2}} \\
\int_{0}^{\hbar \omega_{b}} \frac{\tanh \frac{\beta}{2}\left(\epsilon_{\kappa}^{2}+M^{2}\right)^{1 / 2}}{\left(\epsilon_{\kappa}{ }^{2}+M^{2}\right)^{1 / 2}} d \epsilon_{\kappa} \\
=\ln \frac{\beta \hbar \omega_{b}}{2}-\ln (\pi / 4 \gamma) \\
-M^{2}\left(\frac{1}{\pi k_{\beta} T_{S C}}\right)^{2} 1.052
\end{gathered}
$$

Using the fact that, for low temperature, $\tanh \left(\frac{\hbar \omega_{b}}{2 k_{B} T}\right) \rightarrow 1$,

Where $\gamma$ is the Euler constant having the value $\gamma=$ 1.78 (Hsian) [14] and the last equation can be neglected since $M^{2}$ is very small.

$$
\begin{aligned}
& \text { we can write (48) as, } \\
& I_{1}=\ln \left(1.14 \frac{\hbar \omega_{b}}{k_{B} T_{s c}}\right)
\end{aligned}
$$

Using L' Hospital's rule, it is easy to show that

$$
I_{2}=-\int_{0}^{\hbar \omega_{b}}\left(M^{2} \beta\right) \frac{\operatorname{sech}^{2}\left(\frac{\beta \sqrt{\epsilon_{\kappa}^{2}+M^{2}}}{2}\right)}{2\left(\epsilon_{\kappa}{ }^{2}+M^{2}\right)} d \epsilon_{\kappa}
$$

which can be neglected since $M_{S D W}^{2}$ is very small.

Substituting (49) in (46), we then obtain

$$
\frac{1}{\alpha}=\ln \left(1.14 \frac{\hbar \omega_{b}}{k_{B} T_{C}}\right)
$$

This implies,

$$
T_{C}=\frac{1.14 \hbar \omega_{b}}{k_{B}} \exp \left(-\frac{1}{\alpha}\right),
$$

which can be used to estimate $\exp \left(-\frac{1}{\alpha}\right)$ for $\mathrm{NaFe}_{1-\mathrm{x}} \mathrm{Co}_{\mathrm{x}} \mathrm{As}$, using the experimental value $T_{s c}$ and cut-off energy.

To study how $M$ depends on the magnetic transition temperature $T_{S D W}$, we consider (41).

$$
M=-\alpha_{j} \Delta_{j} \int_{0}^{\hbar \omega_{b} \tanh \frac{\beta}{2}\left(\epsilon_{\kappa}^{2}+\Delta_{j}^{2}(k)\right)^{1 / 2}}{\left(\epsilon_{\kappa}^{2}+\Delta_{j}^{2}(k)\right)^{1 / 2}}^{2} d \epsilon_{\kappa}
$$

Proceeding as before, it is easy to show that,

$$
\begin{aligned}
M=--\alpha_{j} \Delta_{j}(\ln & \left(1.14 \frac{\hbar \omega_{b}}{k_{B} T_{S D W}}\right) \\
& \left.-\Delta_{j}^{2}\left(\frac{1}{\pi k_{\beta} T_{S D W}}\right)^{2} 1.052\right)
\end{aligned}
$$

Neglecting $\Delta_{j}^{2}$

$$
M=-\left(\boldsymbol{\alpha}_{\boldsymbol{j}} \Delta_{\boldsymbol{j}}\right) \ln \left(1.14 \frac{\hbar \omega_{b}}{k_{B} T_{S D W}}\right)
$$

This gives;

$$
\therefore T_{S D W}=\left(\frac{1.14 \hbar \omega_{b}}{k_{B}}\right) \exp \left(\frac{M}{\boldsymbol{\alpha}_{\boldsymbol{j}} \Delta_{\boldsymbol{j}}}\right)
$$
$T_{S D W}$.

We can use (52) to draw the phase diagram for $\mathrm{M}$ and

\section{PAIRING OF SPIN DENSITY WAVE (SDW) AND TRIPLET SUPERCONDUCTIVITY}

In this section we want to drive an expressions for the order parameters of SDW, M, and triplet superconductivity, $\Delta_{\mathbf{S C}}$, as a function of both of them and temperature, and to compare the variation of each with temperature. Still we can use the Hamiltonian given by equation (1), but in this case the superconducting order parameter depends on spin alignment [15] and they can be expressed as;

$$
\begin{aligned}
H=\sum_{p \sigma} \epsilon_{p} a_{p \sigma}^{\dagger} \hat{a}_{p \sigma} & +M \sum_{p}\left(\hat{a}_{p+q \uparrow}^{\dagger} a_{-p \downarrow}+\hat{a}_{-p \downarrow}^{\dagger} a_{p+q \uparrow}\right) \\
& +\Delta_{\mathrm{SC}} \sum_{p}\left(\hat{a}_{p \uparrow}^{\dagger} a_{-p \downarrow}^{\dagger}+\hat{a}_{-p \downarrow} \hat{a}_{p \uparrow}\right)(53)
\end{aligned}
$$

where the superconducting order parameter is given by:

$$
\Delta=\sum_{P}<\hat{a}_{k \sigma}^{\dagger}, \hat{a}^{\dagger}-k \sigma>
$$


We now consider the equation of motion:

$$
\omega \ll \hat{a}_{\kappa \sigma}^{\dagger}, \hat{a}_{-\kappa \sigma}^{\dagger} \gg=\ll\left[\hat{a}_{\kappa \sigma}^{\dagger}, H\right], \hat{a}_{-\kappa \sigma}^{\dagger} \gg
$$

Doing a lot as we did in the above for the commutation and using the assumption $\delta_{\sigma, \downarrow}=1, \delta_{\sigma, \uparrow}=0$ we finally get:

$$
\begin{gathered}
\left(\omega+\epsilon_{\kappa}\right) \ll \hat{a}_{\kappa \downarrow}^{\dagger}, \hat{a}^{\dagger}{ }_{-\kappa \downarrow} \gg=-M \ll \hat{a}^{\dagger}{ }_{\kappa+q \uparrow}, \hat{a}^{\dagger}{ }_{-\kappa \downarrow} \gg-\Delta_{\mathrm{k} \downarrow} \\
\ll \hat{a}_{-k \downarrow}, \hat{a}^{\dagger}{ }_{-\kappa \downarrow} \gg
\end{gathered}
$$

The nesting property of the Fermi surface that expected for low dimensional band structure and attributed to the SDW ordering gives as an expression $\Delta_{-\mathrm{k}}=-\Delta_{\mathrm{k}}$.

Finally:

$$
\left(\omega+\epsilon_{\kappa}\right) \ll \hat{a}_{\kappa \downarrow}^{\dagger}, \hat{a}_{-\kappa \downarrow}^{\dagger} \gg=-\Delta_{\mathrm{k} \downarrow} \ll \hat{a}_{-k \downarrow}, \hat{a}_{-\kappa \downarrow}^{\dagger}
$$

Since we are dealing with only the triplet pair; we can ignore the singlet correlation.

The equation of motion for correlation in RHS of (57) is written as:

$$
\begin{array}{r}
\left(\omega-\epsilon_{-\kappa}\right) \ll \hat{a}_{-k \downarrow}, \hat{a}_{-k \downarrow}^{\dagger} \gg=1+M \ll, \hat{a}_{-k+q \uparrow}, \hat{a}^{\dagger}{ }_{-\kappa \downarrow} \\
\gg-\Delta_{\mathrm{k} \downarrow} \ll \hat{a}^{\dagger}{ }_{k \downarrow}, \hat{a}^{\dagger}{ }_{-\kappa \downarrow} \gg(58)
\end{array}
$$

and

$$
\begin{gathered}
\left(\omega-\epsilon_{-\kappa+q}\right) \ll \hat{a}_{-k+q \uparrow}, \hat{a}_{-k \downarrow}^{\dagger} \gg=M \ll \hat{a}_{-k \downarrow}, \hat{a}^{\dagger}{ }_{-\kappa \downarrow} \\
\text { (59) }
\end{gathered}
$$

This can be rewritten, after solving the commutation relation and removing the singlet pair.

From eq.(58) and (59), we will get;

$$
\begin{aligned}
\left(\omega-\epsilon_{-\kappa}\right) \ll \hat{a}_{-k \downarrow}, \hat{a}^{\dagger}{ }_{-k \downarrow} \gg & =1+\frac{M^{2}}{\left(\omega-\epsilon_{\kappa+q}\right)} \ll \hat{a}_{-k \downarrow}, \hat{a}^{\dagger}{ }_{-k \downarrow} \\
\gg-\Delta_{k \downarrow} \ll & \ll \hat{a}^{\dagger}{ }_{\kappa \downarrow}, \hat{a}^{\dagger}{ }_{-\kappa \downarrow} \gg(60)
\end{aligned}
$$

With help of eq.(60) and eq.(57):

$$
\frac{X Y R-X M^{2}-R \Delta_{K \downarrow}^{2}}{Y R-M^{2}} \ll \hat{a}^{\dagger}{ }_{\kappa \downarrow}, \hat{a}^{\dagger}{ }_{-\kappa \downarrow} \gg=\frac{-\Delta_{K \downarrow}}{Y R-M^{2}}
$$

This can be written as:

$$
\ll \hat{a}^{\dagger}{ }_{\kappa \downarrow}, \hat{a}^{\dagger}{ }_{-\kappa \downarrow} \gg=\frac{-\Delta_{K \downarrow} R}{X Y R-X M^{2}-R \Delta_{K \downarrow}^{2}}
$$

Applying nesting condition $\epsilon_{\kappa}=-\epsilon_{\kappa \pm q}, \epsilon_{-\kappa}=\epsilon_{\kappa \mp q}$ and use approximation, $\epsilon_{\kappa}=\epsilon_{-\kappa}$; eq.(62) becomes:

$$
\ll \hat{a}^{\dagger}{ }_{\kappa \downarrow}, \hat{a}^{\dagger}{ }_{-\kappa \downarrow} \gg=\frac{-\Delta_{K \downarrow}}{X Y-M^{2}-\Delta_{K \downarrow}^{2}}
$$

Using the expression $\omega \rightarrow i \omega_{n}$, eq.(29) and Matsubara's frequency, we can write eq. (63) as:

$$
\Delta_{\mathrm{k}^{\prime} \downarrow}=V \sum_{k} \frac{\Delta_{k \downarrow} \tanh \frac{\left(\sqrt{\epsilon_{\kappa}^{2}+M^{2}+\Delta_{k \downarrow}^{2}}\right)}{2 k_{B} T}}{2 \sqrt{\epsilon_{\kappa}^{2}+M^{2}+\Delta_{k \downarrow}^{2}}}
$$

Where

$$
\Delta_{\mathrm{k}^{\prime} \downarrow}=\frac{\mathrm{V}}{\beta} \sum_{\mathrm{k}, \mathrm{n}} \ll \hat{a}^{\dagger}{ }_{\kappa \downarrow}, \hat{a}_{-\kappa \downarrow}^{\dagger} \gg
$$

$$
\Delta_{\mathrm{k}^{\prime} \downarrow}=\frac{\mathrm{V}}{\beta} \sum_{\mathrm{k}, \mathrm{n}} \frac{\Delta_{K \downarrow}}{\omega_{\mathrm{n}}^{2}+\mathrm{E}_{\mathrm{k}}^{2}}
$$

and $\mathrm{E}_{\mathrm{k}}^{2}=\boldsymbol{\epsilon}_{\boldsymbol{\kappa}}{ }^{2}+M^{2}+\Delta_{\downarrow}^{2}$

By taking an approximation over the superconducting order parameter, such that it is independent of wave vector, finally we get:

$$
1=V \sum_{k} \frac{\tanh \frac{\left(\sqrt{\epsilon_{\kappa}^{2}+M^{2}+\Delta_{\downarrow}^{2}}\right)}{2 k_{B} T}}{2 \sqrt{\epsilon_{\kappa}^{2}+M^{2}+\Delta_{\downarrow}^{2}}}
$$

We now consider the equations of motion for SDW, we can write,

$$
\omega \ll \hat{a}_{\kappa \uparrow}^{\dagger}, \hat{a}_{k-q \downarrow}, \gg=\ll\left[\hat{a}_{\kappa \sigma}^{\dagger}, H\right], \hat{a}^{\dagger}{ }_{-\kappa \sigma} \gg
$$

Doing a lot as we did in the above, we finally get:

$$
\ll \hat{a}^{\dagger}{ }_{\kappa \uparrow}, \hat{a}_{k-q \downarrow}, \gg=\frac{-M}{X Z R-\Delta_{k-q \downarrow}^{2} X-M^{2} R}
$$

So,

$$
1=U \sum_{k} \frac{\tanh \frac{\left(\sqrt{\epsilon^{2}+\Delta_{\downarrow}^{2}+M^{2}}\right)}{2 k_{B} T}}{2 \sqrt{\epsilon^{2}+\Delta_{\downarrow}^{2}+M^{2}}}
$$

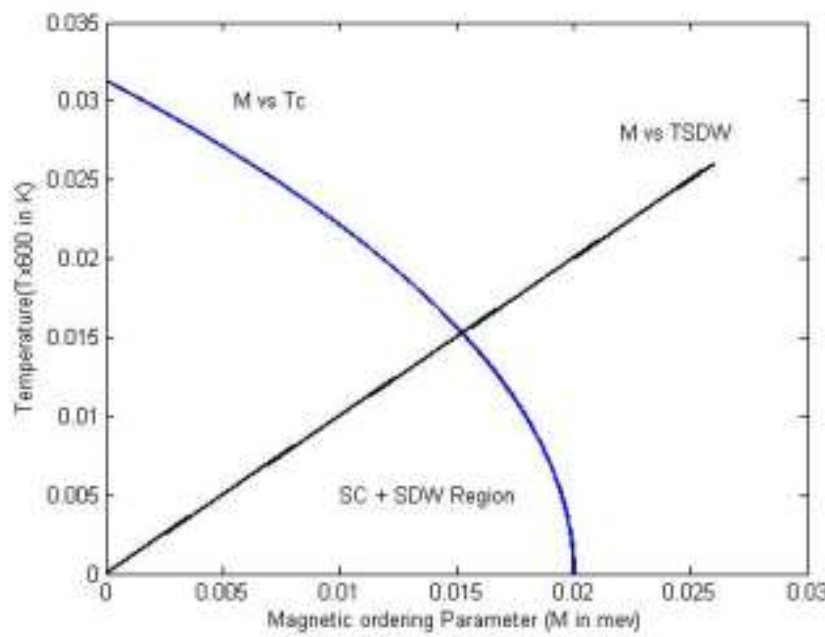

Figure. 1 Co-existence of superconductivity and spin density wave (SDW) in $\mathrm{NaFe}_{1-\mathrm{x}} \mathrm{Co}_{\mathrm{x}} \mathrm{As}$.

\section{RESULTS AND CONCLUSION}

In Fig. 1 we have presented the theoretical curve of the magnetic order parameter $\mathrm{M}$ as a function of the superconducting temperature $\mathrm{T}_{\mathrm{C}}$. For this purpose, we have used (45) which have been numerically solved using the relevant parameters for $\mathrm{NaFe}_{1-\mathrm{x}} \mathrm{Co}_{\mathrm{x}} \mathrm{As}$. In the same figure, we have also plotted the curve of $\mathrm{M}$ as a function of $\mathrm{T}_{\mathrm{SDW}}$, using (52). This curve is found to be almost linear up to the experimental value of $\mathrm{T}_{\mathrm{SDW}}=18 \mathrm{k}$ for $\mathrm{NaFe}_{1-\mathrm{x}} \mathrm{Co}_{\mathrm{x}} \mathrm{As}$.

From Fig. 1 we observe that $\mathrm{T}_{\mathrm{C}}$ decreases with increase in $\mathrm{M}$, whereas $\mathrm{T}_{\mathrm{SDw}}$ increases with increase in $\mathrm{M}$. The superconducting phases and spin density wave, therefore, 
resist each other. However, the present work shows that there is a small region of temperature, where both the phases may be in existence together which is indicated by $(\mathrm{SC}+\mathrm{SDW})$ in the Figure. Thus using a model Hamiltonian consisting of spin density wave and superconducting part and applying Green's function formalism it is possible to derive an expression which shows the relation of the two order parameters and their variation with temperature. This has been done both for singlet and triplet phases of superconductivity coexisting with spin density wave. In the absence of spin density wave the expression for both singlet and triplet cases reduces to the well known BCS result. Our study explicitly shows that superconductivity and spin density wave truly coexist in $\mathrm{NaFe}_{1-\mathrm{x}} \mathrm{Co}_{\mathrm{x}} \mathrm{As}$.

\section{REFERENCES}

[1] Watanabe, T., Yanagi, H., Kamiya, T., Kamihara, Y., Hiramatsu, H., Hirano, M. and Hosono, H, Inorg. Chem. 46, 7719 (2007).

[2] Tegel, M., Bichler, D., and Johrendt, D. Solid State Sci. 10, 193 (2008).

[3] Kamihara, Y., Watanabe, Hirano, T., and Hosono, H. 2008. Iron-Based Layered Superconductor $\mathrm{La}[\mathrm{O} 1-\mathrm{xFx}]$ FeAs $(\mathrm{x}=0.05-0.12)$ with $\mathrm{Tc}=26 \mathrm{~K}$. Journal of the American Chemical Society, 130, 3296-3297.

[4] Chen, G.F., Li, Z., Wu, D., Li, G., Hu, W.Z., Dong, J., Zheng, P., Luo, J.L. and Wang, N.L. 2008. Superconductivity at $41^{\circ} \mathrm{K}$ and Its Competition with Spin-Density-Wave Instability in Layered $\mathrm{CeO1-xFxFeAs.} \mathrm{Physical} \mathrm{Review} \mathrm{Letters,}$

[5] Ren, Z.A., Lu, W., Yang, J., Yi, W., Shen, X.L., Li, Z.C., Che, G.C., Dong, X.L., Sun, L.L., Zhou, F. and Zhao, Z.X.2008. Superconductivity at $55 \mathrm{~K}$ in Iron-
Based F-Doped Layered Quaternary Compound $\mathrm{Sm}[\mathrm{O} 1-\mathrm{xFx}] \mathrm{Fe} A s$. Chinese Physics Letters, 25, 2215.

[6] Kenji, I., Yusuke, N. and Hideo H. 2009. To What Extent Iron-Pnictide New Superconductors Have Been Clarified. Journal of the Physical Society of Japan, 78, ArticleID:062001.http://dx.doi.org/10.1143/JPSJ.78.062 01

[7] Kordyuk, A.A. 2012. Iron-Based Superconductors: Magnetism, Superconductivity, and Electronic Structure. Low Temperature Physics, 38, 1119-1134.

[8] M. Putti et al., Supercond. Sci. Technol. 23, 034003 2010.

[9] Patel et al., Appl. Phys. Lett. 94, 0825082009.

[10] Cai, P. et al. Visualizing the microscopic coexistence of spin density wave and superconductivity in underdoped NaFe1_xCoxAs. Nat. Commun. 4:1596 doi: 10.1038/ncomms2592 (2013).

[11] Marianne Rotter, Marcus Tegel and Dirk Johrendt, 2008. Superconductivity at $38 \mathrm{~K}$ in the iron arsenide (Ba1-xKx)Fe2As2. arXiv:0805.4630v1 [cond-mat.suprcon]

[12] Digor D. F. et.al., Moldavian Journal of the Physical Sciences, Vol.4, No. 4 (2005).

[13] Zubarev, D. N., 1960. Double-time green functions in statistical physics.usp. Fiz. Nauk. Sssr 71:7; Translation: Sov. Phys. Usp. 3, 320-345

[14] Hsian, P. C., 2011. Robust based band reed solomon detection over Power line channel. J.of Engg. Sc. and Tech; 6(1), $69-81$.

[15] Zhou, Y., and Gong, C. D. Europhys. Lett., 74, No. 1, pp. 145150 (2006). 